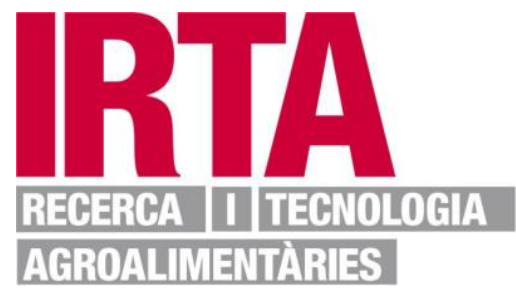

This document is a postprint version of an article published in Meat Science OElsevier after peer review. To access the final edited and published work see https://doi.org/10.1016/j.meatsci.2019.04.018 


\title{
1 Automatic ham classification method based on support vector 2 machine model increases accuracy and benefits compared to manual classification
}

Gerard Masferrer $^{\mathrm{a}, \mathrm{b}}$, Ricard Carreras ${ }^{\mathrm{b}}$, Maria Font-i-Furnols ${ }^{\mathrm{c}}$, Marina Gispert ${ }^{\mathrm{c}}$, Moises Serra ${ }^{\mathrm{a}}$, Pere Marti-Puig ${ }^{\mathrm{a}}$

anformation and Digital Technology Department, UVic-UCC, Vic (Barcelona)

${ }^{b}$ Mafrica.SA, Paratge Can Canals Nou, S/N 08250 Sant Joan de Vilatorrada (BCN)

${ }^{c}$ IRTA - Product Quality, 17121 Monells, Girona, Spain

\begin{abstract}
The thickness of the subcutaneous fat (SFT) is a very important parameter in the ham, since determines the process the ham will be submitted. This study compares two methods to predict the SFT in slaughter line: an automatic system using an SVM model (Support Vector Machine) and a manual measurement of the fat carried out by an experienced operator, in terms of accuracy and economic benefit. These two methods were compared to the golden standard obtained by measuring SFT with a ruler in a sample of 400 hams equally distributed within each SFT class. The results show that the SFT prediction made by the SVM model achieves an accuracy of $75.3 \%$, which represents an improvement of 5.5\% compared to the manual measurement. Regarding economic benefits, SVM model can increase them between 12-17\%. It can be concluded that the classification using SVM is more accurate than the one performed manually with an increase of the economic benefit for sorting.
\end{abstract}

Keywords dry-cured hams; ham-fat grading; subcutaneous fat thickness; pattern recognition; sorting

\section{Introduction}

The thickness of subcutaneous fat (SFT) is one of the most critical parameters in hams for several reasons. Indeed, the thickness of the fat usually determines the process to which the ham will be subjected: dry-curing, cooking or the processing of the raw meat (Bosi, Russo, \& Paolo, 2004). Moreover, SFT is particularly significant in the dry-curing process, as it is one of the critical factors determining the final quality of the product (Candek-Potokar \& Skrlep, 2012). The SFT and the weight mostly determine the amount of salt and other ingredients necessary for the drycuring process (Škrlep et al., 2016) and the curing time (Buscailhon, Gandemer, \& Monin, 1994; 
Marriott, Graham, \& Claus, 1992; Toldrá \& Flores, 1998; Toldrá, Flores, \& Sanz, 1997). Besides, SFT measure is essential to determine the yield in the production of raw meat, which in turn determines the lean percentage of the piece.

It is possible to measure SFT once the green ham has been shaped, however it would be interesting to estimate SFT measure on-line in order to classify the ham before being processed (Masferrer et al. 2018). Optimization of the industrial processes in slaughterhouses is essential to become more competitive. A correct classification of the carcass and the ham on-line can lead to a substantial increase in the slaughterhouse yield. Indeed, it reduces the number of reprocessed primal cuts and allows the linearization of the processes in the cutting plant according to the characteristics of the batches, such as SFT, weight of the ham or breed.

For the online classification of the carcasses and, consequently, the hams, the carcass weight and the lean meat percentage (LMP) are usually used, as they are mandatory data that slaughterhouses must measure according to the Commission Delegated Regulation (EU) 2017/1182. Although, the results obtained using those parameters are positively correlated with ham SFT (Gispert et al., 2007; Pulkrábek, Pavlík, \& Valis, 2006), there is significant room for improvement. On the other hand, there are slaughterhouses where hams are specifically classified according to their own characteristics, such as SFT or LMP of the ham. Furthermore, some slaughterhouses use online predictors obtained from automatic or semi-automatic devices (Font i Furnols \& Gispert, 2009), such as AutoFom and Fat-o-Meater or manual classification of the ham measuring the SFT employing a pattern according to ZP (Zwei-Punkte Messverfahren) measure or similar (Fonti-Furnols et al., 2016).

Pattern recognition systems are widely used in many fields (Bishop, 2006; Jain, Duin, \& Jianchang Mao, 2000). One of the most commonly used algorithms is the Support Vector Machine (SVM). The SVM algorithm is based on finding a hyperplane of separation between different categories. These type of algorithms allow making predictions of categories, in our case the categories based on the SFT of the ham. Those algorithms could be a useful tool in the meat industry, as the amount of data collected from the entire production chain, including the farm and the slaughterhouse, can be significant.

Another relevant factor is the speed of the classification process, as the automatic algorithms, in addition to replacing manual work, allow sorting at high rates compared with manual classification. The objective of this study was to compare a SVM classification algorithm with a manual classification system, commonly used in commercial slaughterhouses, in order to classify hams according to their SFT. The SVM algorithm employs a middle Gaussian core, the best model obtained in Masferrer et al. (2018), which is trained with intrinsic data of the pigs (sex, weight and breed) and data predicted by AutoFom-III (Frontmatec Smørum A/S, Herlev, Denmark) 
(Brøndum, Egebo, Agerskov, \& Busk, 1998). Furthermore, it is also an objective of this work to evaluate the economic effect of the accuracy on ham classification method for the meat industry.

\section{Material and Methods}

\subsection{Animals and facilities}

A total of 400 hams were selected from pigs slaughtered in the $13^{\text {th }}$ of March 2018 at a commercial slaughterhouse (MAFRICA S.A.) located in Sant Joan de Vilatorrada, Catalonia, Spain (see section 2.2). The animals selected included three different genetic lines: (Large White $\times$ Landrace) $\times$ Piétrain, (Large White x Landrace) x Duroc and (Large White $\mathrm{x}$ Landrace) $\mathrm{x}$ (Duroc $\mathrm{x}$ Landrace). Animals came from farms, all of them less than $200 \mathrm{~km}$ far from the slaughterhouse and pigs were transported using trucks in groups of between 80 and 220 animals. Once in the slaughterhouse pigs rested into lairage pens between 2 and 4 hours before being slaughtered.

Pigs were slaughtered after stunning with $\mathrm{CO}_{2}(90 \%)$ for 2 min. After pig scalding process, pigs were totally scanned using the ultrasound AutoFom-III system. Then pigs were eviscerated and automatically split according to standard commercial procedures using a robot. After that, the two half-carcasses were weighted and an experienced operator visually determined the sex of the pig (female, entire male or castrated male) and classified the half carcass as described in Masferrer et al. (2018), in order to normalize the classification. The left half carcass was always used to avoid possible errors produced by the robot cut deviation. For the Manual Classification (HC_M), SFT was measured with a ruler according to minimal fat depth over muscle gluteus medius. The following SFT thresholds were used: class $\mathrm{HC} 1:<9 \mathrm{~mm}$; class HC2: between 9-12 mm; class HC3: between 13-19 mm and class HC4: > $19 \mathrm{~mm}$. The thresholds used were determined by commercial requirements of the slaughterhouse where this study was carried out. HC_M was performed by one experienced operator who usually does the classification in the line.

With the measures obtained with Autofom III and including information about sex, breed and warm carcass weight (see Table.1), the ham class predicted by SVM algorithm (HC_SVM) was obtained (Masferrer et al, 2018).

Table 1. The eleven predictors used as the input of automatic classification system (SVM)

\begin{tabular}{ll}
\hline Predictor & Description \\
\hline LMP & $\begin{array}{l}\text { Lean Meat Percentage } \\
\text { According to the official formula, the subcutaneous fat thickness at } 60 \mathrm{~mm} \text { in the mid-line } \\
\text { F34 }\end{array}$ \\
between the $3^{\text {rd }}$ and the $4^{\text {th }}$ last rib. (mm) \\
According to the official formula, muscle thickness at $60 \mathrm{~mm}$ in the mid-line between the $3^{\text {rd }}$ and \\
the $4^{\text {th }}$ last rib. $(\mathrm{mm})$ \\
The minimum subcutaneous fat plus skin thickness measured with a ruler over the muscle
\end{tabular}




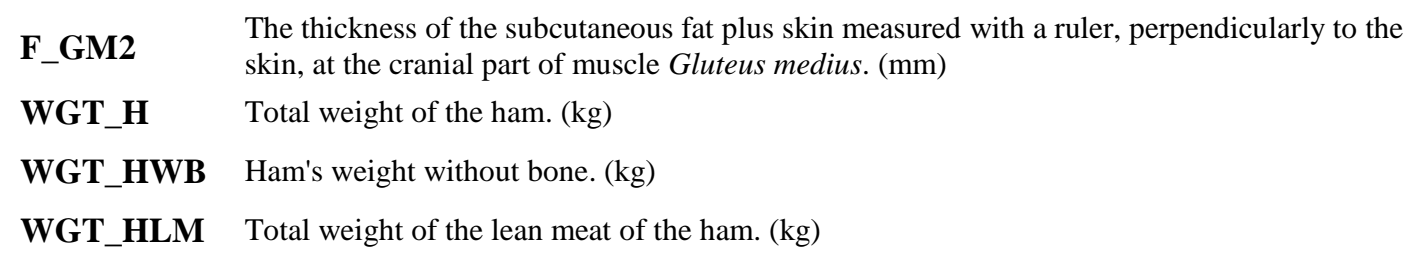

\begin{tabular}{ll}
\hline SEX & Sex of animals (females, entire males and castrated males) \\
BREED & $\begin{array}{l}\text { Crossbreed ((Large White } x \text { Landrace) } x \text { Pietrain, , (Large White x Landrace) x Duroc, and } \\
\text { (Large White x Landrace }) \text { (Duroc x Landrace) }\end{array}$ \\
WGT & Warm carcass weight $(\mathrm{kg})$ \\
\hline
\end{tabular}

\subsection{Ham shaping}

97

Once the carcasses were pre-trimmed in the cutting room, they were refrigerated for 24 hours in a chilling room. When carcasses reached approximately $4^{\circ} \mathrm{C}$ and the ham was extracted and processed to give the final shape and classified according to customer specifications (weight and SFT). Final shape process consisted of removing the tail, rounding off the bottom of the ham and lifting the leg (Fig. 1).

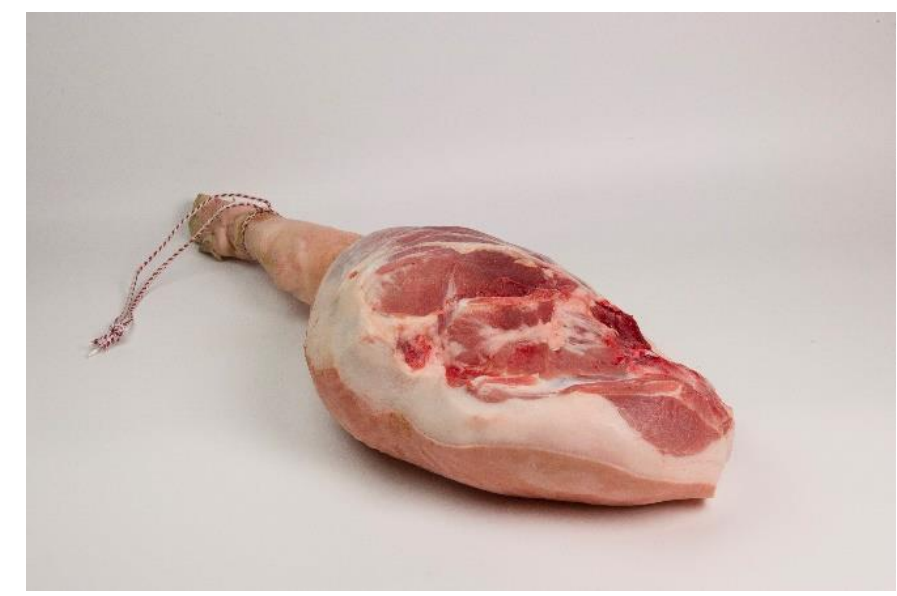

Fig. 1. Ham after final shape process

After the final shape process, 400 hams, one from each carcass were selected according to the SFT measured at that moment. To obtain this parameter an operator employed a ruler to measure the minimal SFT of the ham located in the central part of the muscle gluteus medius, perpendicular to the skin (Golden Standard measure), as shown in Fig.2. Those 400 hams were equally distributed in four SFT classes of 100 samples: HC1: < $9 \mathrm{~mm}$; class HC2: between 9-12 mm; class HC3: between 13-19 mm and class HC4: > $19 \mathrm{~mm}$ ). Hams were randomly measured until 100 samples were obtained for each category. When a class reaches 100 samples, no more hams were selected for that class. The measures obtained using this methodology were necessary to create the Ham Classification used as Golden Standard, (HC_GS) in order to assess the accuracy of the prediction of HC_M and HC_SVM classifications. 


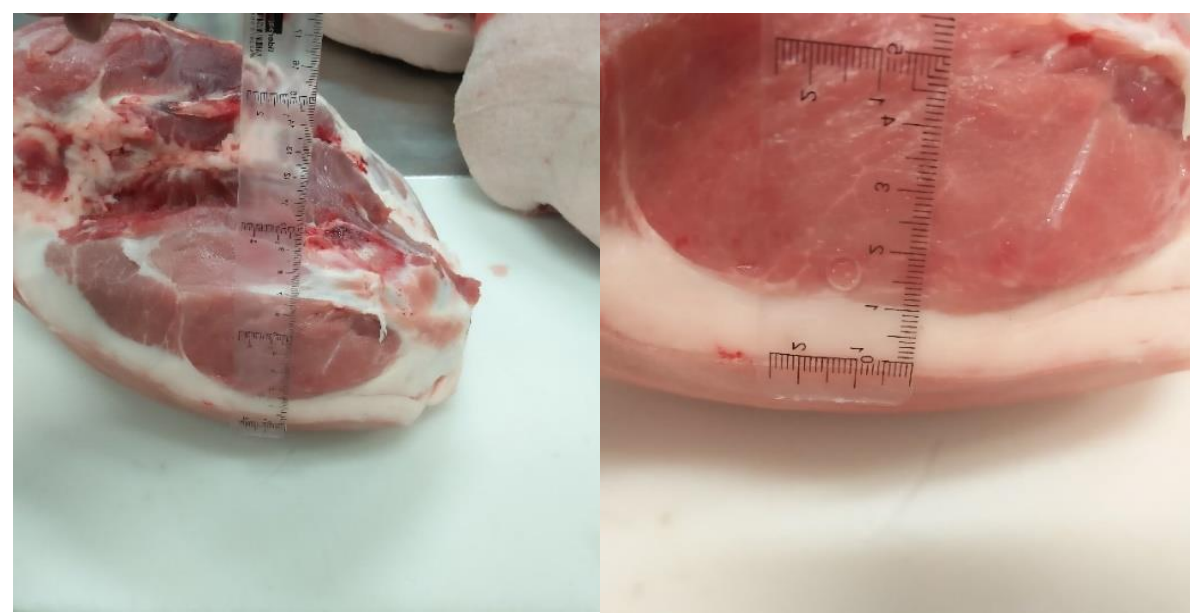

115 Fig. 2. Representation of the section and the ruler used to measure the minimal SFT of the ham located in the muscle 116 gluteus medius.

117 The characteristics of the pigs included in this work are presented in Table 2. It shows the mean and the standard deviation of the warm carcass weight $(\mathrm{kg})$ and the fat thickness $(\mathrm{mm})$ of the 119 evaluated carcasses according to breed and sex. Fat thickness parameter is given by the ultrasound 120 AutoFom-III system and it corresponds to the parameter F34, which is described as the fat 121 thickness at $60 \mathrm{~mm}$ in the mid-line between the $3^{\text {rd }}$ and the $4^{\text {th }}$ last ribs.

Table 2. Warm carcass weight and fat thickness at $60 \mathrm{~mm}$ in the mid-line between the $3^{\text {rd }}$ and the $4^{\text {th }}$ last rib of 400 carcasses according to breed and sex.

\begin{tabular}{llll}
\hline BREED & $\mathbf{n}$ & $\begin{array}{l}\text { WEIGHT } \\
(\text { mean } \pm \text { s.d; } \mathbf{k g})\end{array}$ & $\begin{array}{l}\text { FAT THICKNESS } \\
(\mathbf{m e a n} \pm \text { s.d; } \mathbf{~ m m})\end{array}$ \\
\hline (Large White $\times$ Landrace) $\times$ Piétrain & 218 & $89.95 \pm 8.12$ & $15.13 \pm 4.38$ \\
(Large White x Landrace) x Duroc & 139 & $93.19 \pm 9.46$ & $23.71 \pm 5.11$ \\
(Large White x Landrace) x (Duroc x Landrace) & 43 & $94.48 \pm 8.68$ & $22.11 \pm 9.36$ \\
\hline SEX & & & \\
\hline Female & 205 & $91.95 \pm 8.88$ & $16.25 \pm 4.10$ \\
Castrated males & 150 & $92.32 \pm 9.54$ & $24.49 \pm 6.49$ \\
Entire males & 45 & $87.23 \pm 3.31$ & $11.99 \pm 2.14$ \\
\hline
\end{tabular}

\subsection{Statistical analysis}

The objective of this statistical evaluation was to compare the classification obtained with the manual classification (HC_M) and the automatic classification performed by the SVM, respectively. SVM refers to Gaussian Medium algorithm (HC_SVM), the best model obtained in Masferrer et al. (2018), to classify hams according to SFT. In order to evaluate the prediction of the ham class, the SFT of the finished hams was specifically measured for this study, in order to obtain the Golden Standard Ham Class (HC_GS) as described in the previous section. 
To compare the results obtained with HC_M and HC_SVM a Cohen's kappa coefficient (kvalues) was used to compare the classification performed by HC_M and HC_SVM methods and to compare HC_M and HC_SVM classifications with HC_GS, respectively. The guidelines developed by Landis \& Koch (1977) were used to interpret the k-values: poor agreement $(\mathrm{k}<$ $0.00)$, soft agreement $(\mathrm{k}=0.00-0.20)$, fair agreement $(\mathrm{k}=0.21-0.40)$, moderate agreement $(\mathrm{k}=$ 0.41-0.60), substantial agreement $(\mathrm{k}=0.61-0.80)$ and almost perfect agreement $(\mathrm{k}=0.81-1)$.

The means and variances of SFT measured by HC_GS according to the category assigned by the classification systems (HC_M and HC_SVM) were calculated. T-Test and Bartlett's Test were carried out to assess the mean and the homogeneity of variances, respectively. The significance level was established at $p<0.05$. Descriptive data is presented with means of $\mathrm{mm}$ and the standard error (mean $\pm \mathrm{SE})$.

Moreover, the accuracy of the prediction of HC_M and HC_SVM according with the results of HC_GS was calculated. The accuracy of the prediction is defined as the number of correct predictions divided by the number of total predictions. As a result, two confusion matrices were constructed using a cross-table with the real values (HC_GS) obtained by the "golden standard", and those provided by the operator (HC_M) and by the algorithm (HC_SVM), respectively. The accuracy was also calculated by breed and sex.

In order to obtain additional information (i.e. assess if better results could be obtained using Golden Standard measures to train SVM, instead of using measures obtained with the manual measurement on-line), the SVM was trained with the measures obtained to create the HC_GS. The same predictors were used (see Table 1) but using HC_GS as an independent variable (response). Moreover, the same SVM Medium Gaussian was used, and the training and verification phase was a 5-fold Cross-Validation.

MATLAB, Statistics and Machine Learning Toolbox ${ }^{\mathrm{TM}}$ (Matlab R2018a; The MathWorks, Inc, 1988-2019) have been used to develop and test all the models and algorithms.

Moreover, the economic impact using those classification methods on the slaughterhouse was analysed and compared between the HC_M and HC_SVM. This slaughterhouse slaughters approximately 500,000 pigs/year (8,000-10,000 pigs/week). The distribution of ham classes according to sales data of hams of 2017 was $54 \%$ as $\mathrm{HC} 1,28 \%$ as $\mathrm{HC} 2,13 \%$ as $\mathrm{HC} 3$ and $5 \%$ as HC4. The economic data taken as reference correspond to the slaughterhouse where this study was carried out (MAFRICA S.A.). The economic profit according to increase in price due ham classification has been taken into account. This increase in prices was estimated reflecting an optimistic and pessimistic increased price according to slaughterhouse commercial data. 
increase of price between +0.03 and $+0.10 € / \mathrm{kg}$, hams in category HC3 have an increase of price between +0.12 and $+0.20 € / \mathrm{kg}$ and hams in category HC4 have an increase of price between +0.15 and $0.30 € / \mathrm{kg}$. To estimate the weight of the hams, the average of the warm weight of the carcasses of the population of 2017 (i.e. $87 \mathrm{~kg}$ ) was used, taking into account that the ham represents between 28-30\% of the carcass (Cisneros, Ellis, McKeith, McCaw, \& Fernando, 1996; Gispert et al., 2007).

172

An increase of economic value was only applied when the ham was correctly classified by the predictors. Moreover, when a ham was misclassified, it was considered that the cost of reprocess or reclassify had a similar cost to the increase of price that could be assigned.

\section{Results and discussion}

\subsection{Comparisons of classification methods}

177 The confusion matrices plot as a cross-table are shown in Fig. 3, where the rows correspond to 178 the predicted class (HC_M and HC_SVM, respectively) and the columns correspond to the 179 HC_GS class, that is, the Golden Standard. The diagonal cells correspond to samples that are 180 correctly classified. The off-diagonal cells correspond to incorrectly classified samples. Both the 181 number of samples and the percentage of the total number of samples are shown in each cell. The 182 column on the right of the matrix shows the percentages of all the samples predicted to belong to 183 each class that are correctly (positive predictive value) and incorrectly (false discovery rate) 184 classified. The row at the bottom shows the percentages of all the samples belonging to each class that are correctly (true positive rate) and incorrectly (false negative rate) classified. The cell in the bottom right of the matrix shows the overall accuracy.
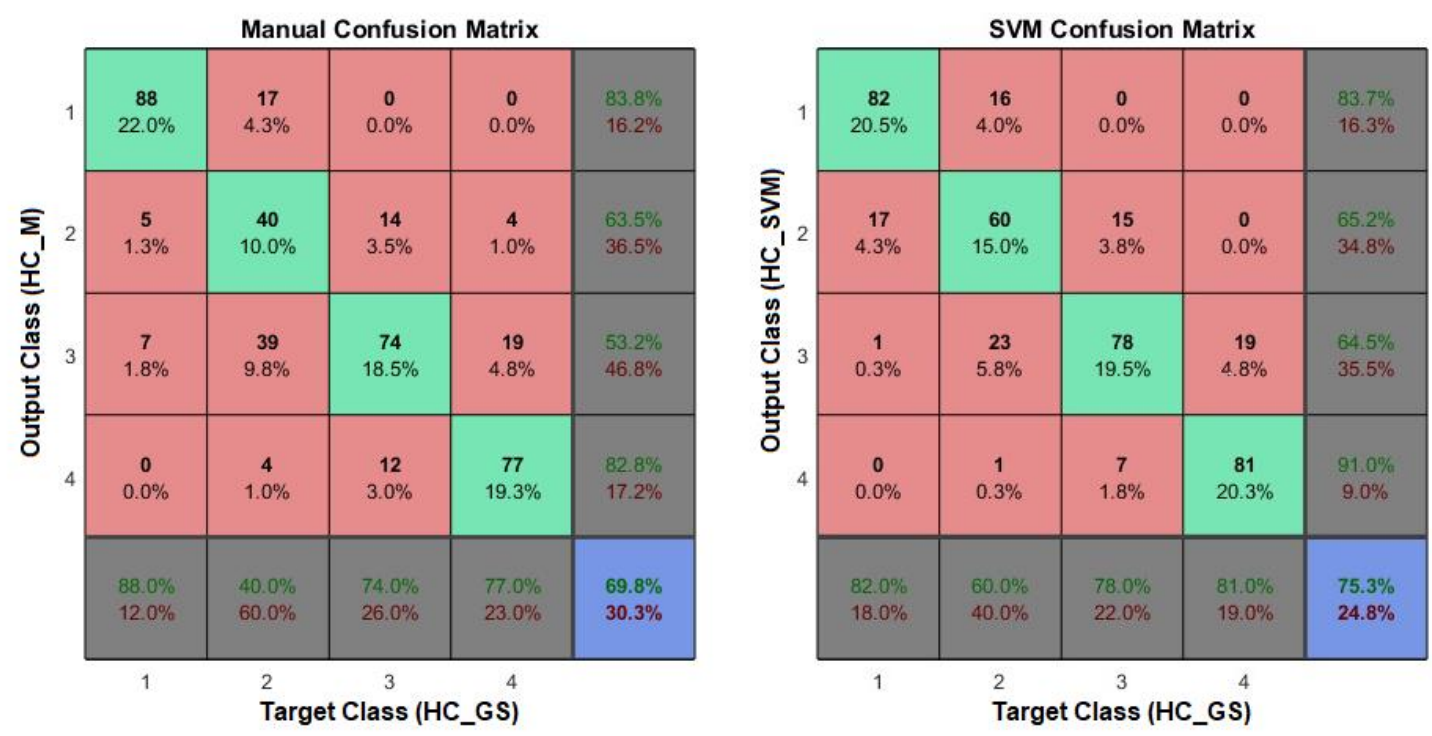

187 
The accuracy of the prediction of HC_SVM was better than the HC_M $(75.3 \%$ and $69.8 \%$, respectively). Indeed, HC_SVM obtained better results in all classes except for HC1, where the HC_M obtained an $88 \%$ of correct predictions compared to the HC_SVM that obtained an $82 \%$. This exception could be related to some Autofom III parameter used in the SVM algorithm. Specifically, the parameter F_GM1 (The minimum subcutaneous fat thickness over the muscle Gluteus medius (mm)) seems to be difficult to measure by AutoFom when SFT is very low. Indeed, Fig. 4 shows that hams with SFT lower than $6 \mathrm{~mm}$ according to HC_GS are overestimated by the F_GM1 parameter, (only eight hams have values below $6 \mathrm{~mm}$ of subcutaneous fat from 32 obtained in HC_GS). Moreover, Fig. 4 shows that F_GM1 estimates the central values of SFT with more precision than extreme values, especially underestimate higher SFT values.

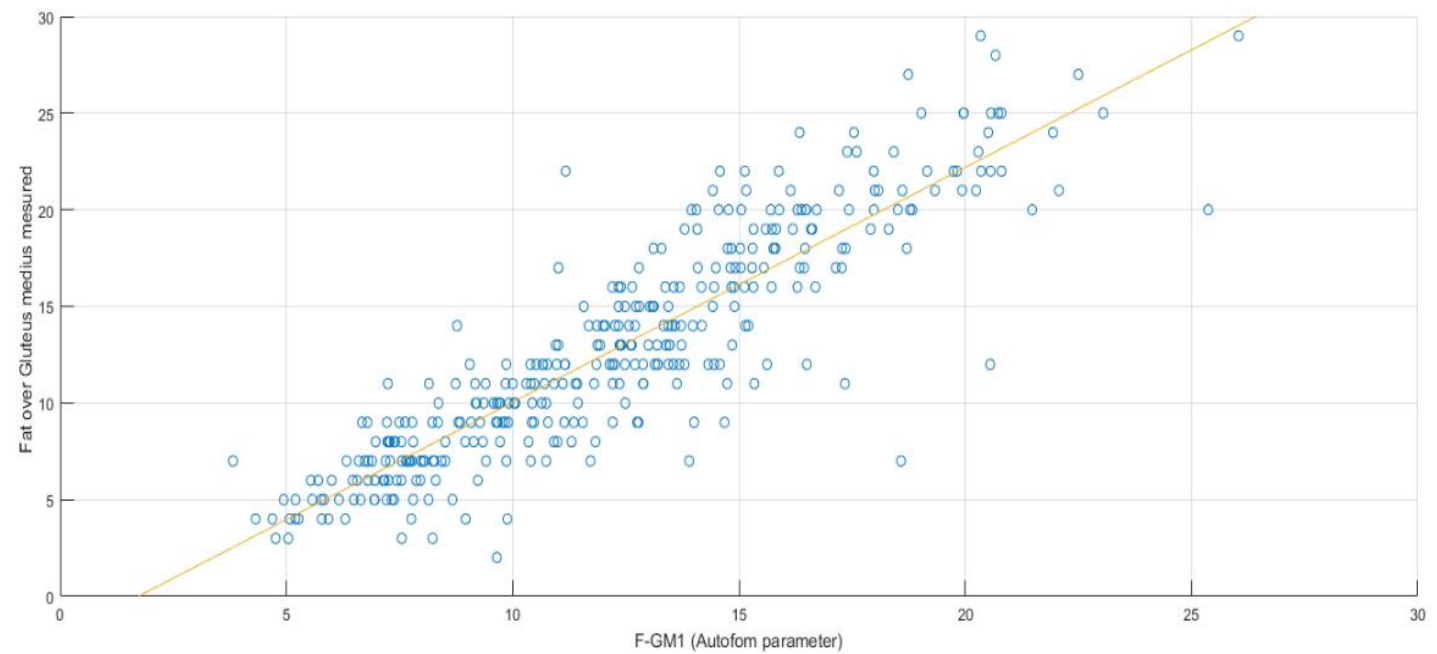

Fig.4. Correlation between Golden Standard measure ( $m m$ of subcutaneous fat thickness of the ham after final shape process) and F-GM1 Autofom III parameter (mm of the minimum subcutaneous fat thickness over the muscle Gluteus medius)

Concerning the remaining $\mathrm{HC} 2, \mathrm{HC} 3$, and $\mathrm{HC} 4$ classes HC_SVM provides better predictions than HC_M improving the accuracy of prediction of $\mathrm{HC} 3$ and $\mathrm{HC} 4$ categories by a $4 \%$ and surprisingly by $20 \%$ in HC2. These results suggest that the operator tended to overestimate the class HC2, with a 9.8\% of hams classified in HC3. This tendency is also shown in Fig. 5 where the mean of the SFT estimated by HC_MC measures is higher than the mean of the SFT estimated by HC_SVM measures $(12.34 \pm 0.65$ HC_M2 and $10.58 \pm 0.25$ in HC_SVM2; t-test $\mathrm{p}=0.007)$. Moreover, it seems that the HC_M classification presented higher standard error within their group compared to HC_SVM classification $(p=0.000)$ (see Fig. 5). Indeed, the HC_M tend to overestimate $(16.9 \%)$ in more cases than underestimate $(13.6 \%)$ while the HC_SVM tend to 
overestimate as well as underestimate (12.5 and $12.6 \%$, respectively). These results suggest that measurement methods could explain those differences. A possible explanation is related to fat state, when the operator measured SFT the carcass was hot and was not compacted as it was subjected vertically. Maybe those differences could be the reason for observing more deviation in HC_M measures (see Fig.5). While SFT was measured by Autofom the carcass, even hot, was compacted because this measure was recorded with the carcass with an horizontal position supported on a surface. Moreover, when the Golden Standard measure was taken the carcass had been cooling for 24 hours and the fat was compacted because of low temperatures. Differences between HC_GS and the two other classification methodologies, HC_M and HC_SVM, could also be related to fatty acid profile. According to St. John et al. (1987) and Warnants, Van Oeckel, \& Boucqué (1996), as more content of unsaturated fatty acids more decreases fat firmness and is softer than fat with more content of saturated fatty acids which may affect the thickness of the fat. Although less fatty hams tend to be more unsaturated (Ruiz-Carrascal, Ventanas, Cava, Andrés, \& García, 2000), contrary to what would be expected, fewer differences were found between HC_M and HC_SVM regarding less fat categories, i.e. HC1 and HC2. This result suggests that the effect on the softness of the fat, due to saturated fatty acid content, is less appreciable in less fat categories because of the lower content of fat.

Comparing the results showed on the right column of both matrices suggest that predictions of hams classified in categories HC3 and HC4 obtain better results in HC_SVM than in HC_M (64.5\% vs. $53.2 \%$ in $\mathrm{HC} 3$ and $91.0 \%$ vs. $82.8 \%$ in $\mathrm{HC} 4$, respectively). While the results of the predictions of $\mathrm{HC} 1$ and $\mathrm{HC} 2$ are similar between HC_M and HC_SVM. These results have an impact on the economic benefits, since the categories HC3 and HC4 have a higher economic value than the other categories. These hams are intended for dry-curing processes and it is especially important to increase the percentage of true positives.

Comparing the incorrectly predicted samples between HC_M and HC_SVM is interesting to observe the dispersion of those incorrected samples. While the HC_SVM had the $0.6 \%$ of the incorrect samples not distributed to neighbouring classes, this result increased to $3.8 \%$ in the case of the HC_M. Indeed, this dispersion is also showed in Fig.5 where the standard error is presented for each class in both ham classifications (HC_M and HC_SVM). These cases can lead to unexpected production line problems that force the ham to be reprocessed offline, or being processed inefficiently. Usually, a certain percentage of hams are expected to be destined to neighbouring categories, but they are processed in a similar way, for example, HC3 or HC4 hams are usually destined for dry-curing processes while $\mathrm{HC} 1$ or $\mathrm{HC} 2$ hams are usually deboned to produce raw meat. 
kappa was used to take into account the possibility that the classification is produced by chance.

250 Comparing the classification carried out by HC_M and HC_SVM, a moderate almost substantial agreement was found $(\mathrm{k}=0.596)$. Although it is a good result, the agreement between the two classifications methods could be expected to be slightly higher, as the HC_SVM is a method created to emulate HC_M. Perhaps this result could be explained due to the number of samples used. In this study only 400 hams were analysed due to technical reasons (specifically to obtain the Golden Standard measures). On the other hand, the SVM algorithm used in the present study (HC_SVM) was trained with more than 30000 obtained samples, being more robust.

257

Moreover, when comparing both classification methods with the HC_GS, a substantial agreement $(\mathrm{k}=0.670)$ was found for the HC_SVM method and a moderate almost substantial agreement $(\mathrm{k}$ = 0.597) was obtained for the HC_M. According to these results, it seems that the correct classifications with HC_SVM were slightly higher than with HC_M. Moreover, Fig.5 shows the mean and standard error in mm of SFT of the hams over the muscle gluteus medius calculated by the Golden Standard method for each class (HC1, HC2, HC3 and HC4) and for both methods (HC_M and HC_SVM). Indeed, this figure shows that the standard error of the measures obtained by HC_SVM was lower than the standard error of the values obtained by HC_M. And the results of Bartlett's test show a significant difference on variance between methods in HC2 and HC3 ( $p=$ 0.000 and $p=0.038$, respectively).

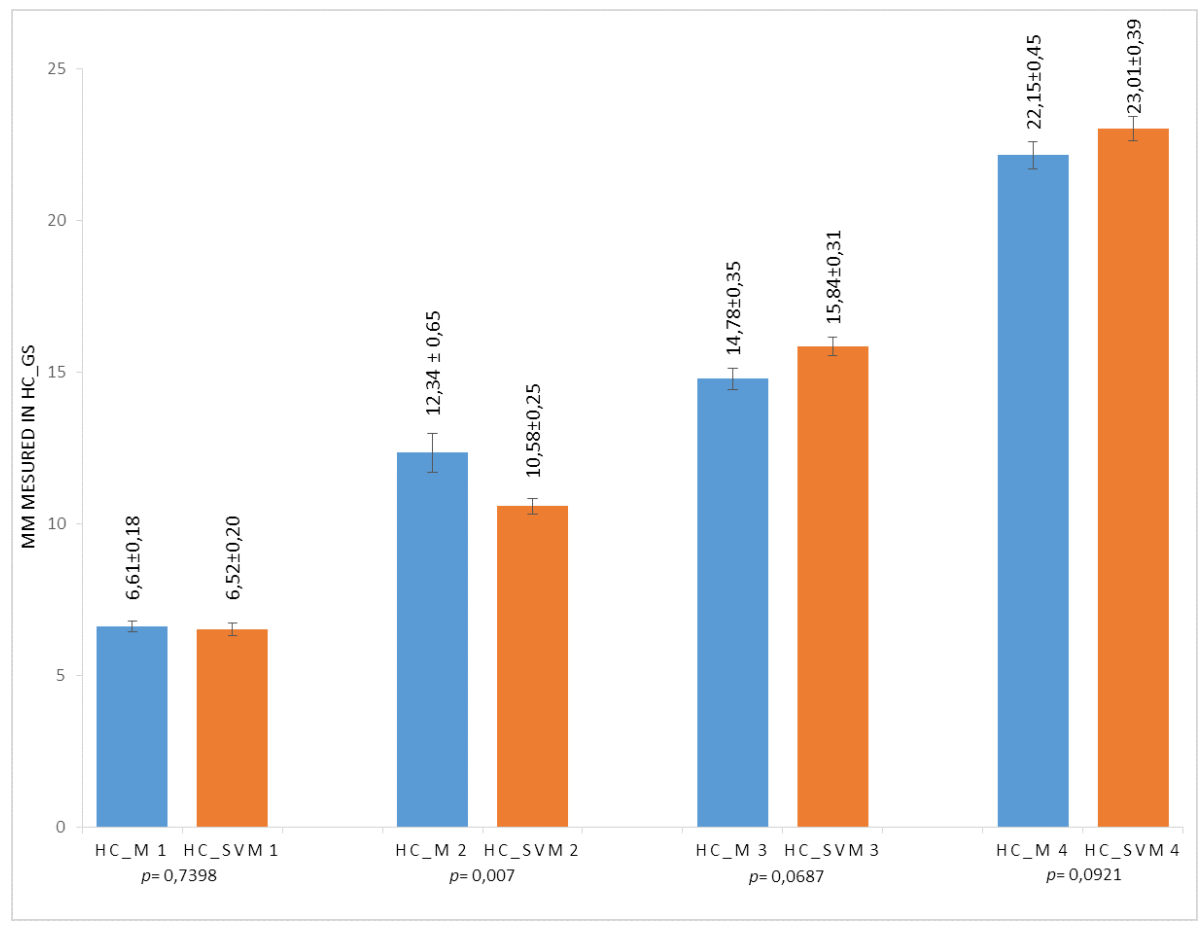

Fig.5 Subcutaneous fat thickness of hams over muscle gluteus medius (mean \pm SE mm) measured by HC_GS according to the category assigned by the classification system HC_M (left columns) and HC_SVM (right columns). At the bottom of columns the p-value of two-samples T-test between HC_M and H_SVM in each ham class. 
271 Other factors may explain why HC_SVM obtained better results than HC_M. One of them is the

272 probably operator fatigue, according to Font-i-Furnols et al. (2016) and Olsen et al. (2007),

273 process repeatability or overexposure to a same class tends to lead to errors in classification (e.g.

274 after classifying as HC4 a large number of hams, hams with slightly less fat tend to be classified

275 into lower categories). Moreover, the cutting process of the carcasses usually obtain asymmetries

276 in the two half-carcasses hindering the process of classification (Nissen et al., 2006). These factors

277 could also affect the HC_GS measures. However, the HC_M measures were obtained on-line,

278 with the speed of the production chain, while HC_GS was done out-line, without any speed chain.

279 These factors suggest that using automatic algorithms with all information available and with a

280 proper training phase it is possible to obtain better predictions of SFT of hams and therefore

281 improve its classification.

Table 3. Percentage of correct, overestimate and underestimate classifications, between Manual prediction (HC_M) and automatic system (HC_SVM) from 400 hams according to breed and sex.

284

285

286

287

288

289

290

291

292

293

294

295

296

297

298

\begin{tabular}{|c|c|c|c|c|c|c|}
\hline & \multicolumn{3}{|c|}{ HC_M } & \multicolumn{3}{|c|}{ HC_SVM } \\
\hline & Correct $^{\mathrm{a}}$ & Over $^{b}$ & Under $^{\mathrm{c}}$ & Correct $^{\mathrm{a}}$ & Over $^{\text {b }}$ & Under $^{c}$ \\
\hline \multicolumn{7}{|l|}{$\overline{S E X}$} \\
\hline Female & $63 \%$ & $23 \%$ & $14 \%$ & $71 \%$ & $15 \%$ & $15 \%$ \\
\hline Castrated males & $73 \%$ & $13 \%$ & $15 \%$ & $77 \%$ & $12 \%$ & $11 \%$ \\
\hline Entire males & $91 \%$ & $2 \%$ & $7 \%$ & $89 \%$ & $2 \%$ & $9 \%$ \\
\hline \multicolumn{7}{|l|}{ BREED } \\
\hline (Large White $\mathrm{x}$ Landrace) $\mathrm{x}$ Duroc & $69 \%$ & $17 \%$ & $14 \%$ & $78 \%$ & $14 \%$ & $9 \%$ \\
\hline $\begin{array}{l}\text { (Large White } \mathrm{x} \text { Landrace) } \mathrm{x} \text { (Duroc } \mathrm{x} \\
\text { Landrace) }\end{array}$ & $67 \%$ & $19 \%$ & $14 \%$ & $79 \%$ & $12 \%$ & $9 \%$ \\
\hline (Large White $\times$ Landrace $) \times$ Pietrain & $71 \%$ & $16 \%$ & $13 \%$ & $73 \%$ & $11 \%$ & $16 \%$ \\
\hline
\end{tabular}

${ }^{\mathrm{a}}$ Correct, ${ }^{\mathrm{b}}$ Over Overestimate, ${ }^{\mathrm{c}}$ Under Underestimate

Table 3 shows the results of the classification of the two assessed methods. The results are shown in percentage and distributed according to successes, overestimated and underestimated categories. Those classifications are showed according to sex, at the top of the table, and according to breed, at the bottom of the table.

The results according to sex show that the HC_SVM obtained better results and the number of overestimate measures of castrated males and females was lower than in HC_M. Moreover, in both methods, the percentage of success in entire males was very high with a percentage of $91 \%$ in the case of HC_M and $89 \%$ in the case of HC_SVM. Those results suggest that better predictions are obtained due to entire males have leaner hams with low deviation of the SFT (see Table 2). Consequently, they were easy to predict as HC1.

Instead, regarding breed, HC_SVM obtained better results of overestimation and underestimation in all breeds except for crossbreed Pietrain underestimation, compared to HC_M. Therefore, correct predictions were similar between the two methods in the case of (Large White $\times$ Landrace) $\times$ Pietrain with $71 \%$ and $73 \%$ of correct predictions of HC_M and HC_SVM, respectively. 
Moreover, HC_SVM obtained better results when crossbreed included a Duroc line, compared to

300 HC_M. Indeed correct predictions were a 9\% and a 12\% higher regarding the (Large White $\mathrm{x}$

301 Landrace) $x$ Duroc and the (Large White x Landrace) x (Duroc x Landrace), respectively.

302 These results suggest that the HC_SVM better predicted ham classification than HC_M, 303 especially in the fattest carcasses which mainly included carcasses from females, castrated males 304 and Duroc crosses according to Gispert et al. (2010) and Wood, Enser, Whittington, Moncrieff, 305 \& Kempster (1989). This makes sense since HC_SVM improves mainly in the fatter groups of 306 classification (HC2 to HC4). In contrast, the results obtained from the leaner carcasses are similar 307 between HC_SVM and HC_M, which mainly included entire males and Pietrain crosses. Those 308 results are especially relevant from an economic point of view, as the fattest hams are the ones 309 that can be more valued for drying purposes.

\subsection{Re-training of the algorithm with HC_GS}

311 To assess whether it was possible to improve the HC_SVM algorithm, the SVM algorithm was 312 re-trained using HC_GS as a response variable. As a result of this test, a model (HC_SVM2) was 313 obtained with an accuracy of $75 \%$ and a coefficient $k$, of 0.67 (substantial agreement) was 314 obtained. The results obtained in the HC_SVM2 model do not improve the percentage of success 315 obtained in the classification of HC_SVM obtained in this study. This result suggests that the 316 original SVM algorithm obtained by Masferrer et al (2018) was as good as the re-trained model 317 HC_SVM2 probable because a large amount of data was used in HC_SVM. Although HC_SVM2 318 did not improve on the previous ones, it might be interesting for future work to explore other 319 methods using HC_GS as a continuous variable.

\subsection{Slaughterhouse profit}

The impact of the correct hams classification is shown in Table 3. This table compares the potential benefits of a commercial slaughterhouse in 2017 classifying hams with a manual classification (HC_M) and with an automatic classification using an SVM model (HC_SVM). In order to quantify this potential benefit, an increase in price according to ham category was assigned as described in section 2.3.

Table 3. Comparison of the potential benefits of a year between classifying hams with the manual classification (HC_M) and with the automatic classification using an SVM model (HC_SVM). Data showed in the table is obtained from 2017 and belongs to a commercial slaughterhouse.

\begin{tabular}{ccccccc} 
HC & $\begin{array}{c}\text { Categories } \\
(\boldsymbol{\%})\end{array}$ & Pigs & $\begin{array}{c}\text { HC_M } \\
\text { Correct }\end{array}$ & $\begin{array}{c}\text { HC_M Profit } \\
\text { increase }\end{array}$ & $\begin{array}{c}\text { HC_SVM } \\
\text { Correct }\end{array}$ & HC_SVM Profit increase \\
\hline HC1 & 54 & 269400 & 237072 & $0 €$ & 220908 & $0 €$ \\
HC2 & 28 & 138850 & 55540 & $42.038-140.127 €$ & 83310 & $63.057-210.191 €$
\end{tabular}




\begin{tabular}{ccccccc} 
HC3 & 13 & 66750 & 49395 & $149.548-249.247 €$ & 52065 & $157.632-262.720 €$ \\
HC4 & 5 & 25000 & 19250 & $72.852-145.703 €$ & 20250 & $76.636-153.272 €$ \\
\hline Total & 100 & 500000 & 361257 & $\mathbf{2 6 4 . 4 3 8 - 5 3 5 . 0 7 8 €}$ & 376533 & $\mathbf{2 9 7 . 3 2 5 - 6 2 6 . 1 8 3} €$ \\
\hline
\end{tabular}

330

As expected, as showed in Table 3 better economic results are obtained using HC_SVM than using HC_M. Indeed, there is a difference between 30.000-90.000€, which represents an increase between $12-17 \%$ of the benefits.

Analysing these results according to HC, the HC_SVM obtained a potential benefit of 5\% higher than using the HC_M in categories HC3 and HC4. Moreover, regarding the hams classified in $\mathrm{HC} 2$ category this difference was more than 50\%. As expected, these results are due to the difference in accuracy of prediction in $\mathrm{HC} 2$ category between the two classification methods, being the HC_SVM better than the HC_M, but also because represents the $28 \%$ of all hams.

Furthermore, although it has not been taken into account in the previous economic analysis, the use of the SVM model allows to classify without an operator, saving the costs of production line personnel.

\section{Conclusions}

The results of the present study suggest that the use of automatic pattern recognition algorithms, and in this particular case, the SVM algorithm improves the prediction of the SFT measure and, therefore, the classification of the ham compared to HC_M. In addition, this method could allow the replacement of an operator in the production line, saving personnel costs, allowing faster chain speeds and reducing errors due to the fatigue of the operator. Moreover, it could improve subsequent processes in the cutting line, reducing the number of reprocessed hams and homogenizing batches for dry-curing processes. Consequently, this automatic HC_SVM method is more accurate and economically more beneficial for the meat industry than the manual HC_M method.

\section{Acknowledgements}

"Financial support from the Agency for Management of University and Research Grants (AGAUR) of the Catalan Government to Gerard Masferrer (2015 DI 00011) is grateful". CERCA Programme (Generalitat de Catalunya) is also acknowledged. The present study was supported by Grup Operatiu (Millora integral de la carn de porc a través d'estratègies productives a nivell de granja i eines innovadores, NIRS on-line per la seva classificació, a nivell d'escorxador. 562104820163A.

\section{References}


Bishop, C. M. (2006). Pattern Recognition and Machine Learning. Pattern Recognition (Vol. 4). http://doi.org/10.1117/1.2819119

Bosi, P., Russo, V., \& Paolo, B. (2004). The production of the heavy pig for high quality processed products. J.ANIM.SCI, 3(21), 309-32145. http://doi.org/10.4081/ijas.2004.309

Brøndum, J., Egebo, M., Agerskov, C., \& Busk, H. (1998). On-Line Pork Carcass Grading with the Autofom Ultrasound System. Journal of Animal Science, 76(7), 1859-1868.

Buscailhon, S., Gandemer, G., \& Monin, G. (1994). Time-related changes in intramuscular lipids of French dry-cured ham. Meat Science, 37(2), 245-255. http://doi.org/10.1016/03091740(94)90084-1

Candek-Potokar, M., \& Skrlep, M. (2012). Factors in pig production that impact the quality of dry-cured ham: a review. Animal: An International Journal of Animal Bioscience, 6(2), 327-38. http://doi.org/10.1017/S1751731111001625

Cisneros, F., Ellis, M., McKeith, F. K., McCaw, J., \& Fernando, R. L. (1996). Influence of Slaughter Weight on Growth and Carcass Characteristics, Commercial Cutting and Curing Yields, and Meat Quality of Barrows and Gilts from Two Genotypes. Journal of Animal Science, 74(5), 925-933. http://doi.org/1996.745925

Font-i-Furnols, M., Čandek-Potokar, M., Daumas, G., Gispert, M., Judas, M., \& Seynaeve, M. (2016). Comparison of national ZP equations for lean meat percentage assessment in SEUROP pig classification. Meat Science, 113, 1-8. http://doi.org/10.1016/j.meatsci.2015.11.004

Font i Furnols, M., \& Gispert, M. (2009). Comparison of different devices for predicting the lean meat percentage of pig carcasses. Meat Science, 83(3), 443-446. http://doi.org/10.1016/j.meatsci.2009.06.018

Gispert, M., Àngels Oliver, M., Velarde, A., Suarez, P., Pérez, J., \& Font i Furnols, M. (2010). Carcass and meat quality characteristics of immunocastrated male, surgically castrated male, entire male and female pigs. Meat Science, 85(4), 664-670. http://doi.org/10.1016/j.meatsci.2010.03.021

Gispert, M., Font i Furnols, M., Gil, M., Velarde, A., Diestre, A., Carrión, D., ... Plastow, G. S. (2007). Relationships between carcass quality parameters and genetic types. Meat Science, 77(3), 397-404. http://doi.org/10.1016/j.meatsci.2007.04.006

Jain, A. K., Duin, P. W., \& Jianchang Mao. (2000). Statistical pattern recognition: a review. IEEE Transactions on Pattern Analysis and Machine Intelligence, 22(1), 4-37. http://doi.org/10.1109/34.824819 
Landis, J. R., \& Koch, G. G. (1977). The Measurement of Observer Agreement for Categorical Data. Biometrics, 33(1), 159. http://doi.org/10.2307/2529310

Marriott, N. G., Graham, P. P., \& Claus, J. R. (1992). ACCELERATED DRY CURING OF PORK LEGS (HAMS): A REVIEW. Journal of Muscle Foods, 3(2), 159-168. http://doi.org/10.1111/j.1745-4573.1992.tb00472.x

Masferrer, G., Carreras, R., Font-i-Furnols, M., Gispert, M., Marti-Puig, P., \& Serra, M. (2018). On-line Ham Grading using pattern recognition models based on available data in commercial pig slaughterhouses. Meat Science, 143, 39-45. http://doi.org/10.1016/j.meatsci.2018.04.011

Nissen, P. M., Busk, H., Oksama, M., Seynaeve, M., Gispert, M., Walstra, P., .. Olsen, E. (2006). The estimated accuracy of the EU reference dissection method for pig carcass classification. Meat Science, 73(1), 22-28. http://doi.org/10.1016/J.MEATSCI.2005.10.009

Olsen, E. V., Candek-Potokar, M., Oksama, M., Kien, S., Lisiak, D., \& Busk, H. (2007). On-line measurements in pig carcass classification: Repeatability and variation caused by the operator and the copy of instrument. Meat Science, 75(1), 29-38. http://doi.org/10.1016/j.meatsci.2006.06.011

Pulkrábek, J., Pavlík, J., \& Valis, L. (2006). Pig carcass quality in relation to carcass lean meat proportion. Czech Journal of Animal. Retrieved from http://agriculturejournals.cz/publicFiles/52250.pdf

Ruiz-Carrascal, J., Ventanas, J., Cava, R., Andrés, A. I., \& García, C. (2000). Texture and appearance of dry cured ham as affected by fat content and fatty acid composition. Food Research International, 33(2), 91-95. http://doi.org/10.1016/S0963-9969(99)00153-2

Škrlep, M., Čandek-Potokar, M., Lukač, N. B., Povše, M. P., Pugliese, C., Labussière, E., \& Flores, M. (2016). Comparison of entire male and immunocastrated pigs for dry-cured ham production under two salting regimes. Meat Science, 111, 27-37. http://doi.org/10.1016/j.meatsci.2015.08.010

St. John, L. C., Young, C. R., Knabe, D. A., Thompson, L. D., Schelling, G. T., Grundy, S. M., $\&$ Smith, S. B. (1987). Fatty Acid Profiles and Sensory and Carcass Traits of Tissues from Steers and Swine Fed an Elevated Monounsaturated Fat Diet. Journal of Animal Science, 64(5), 1441-1447. http://doi.org/10.2527/jas1987.6451441x

Toldrá, F., \& Flores, M. (1998). The Role of Muscle Proteases and Lipases in Flavor Development During the Processing of Dry-Cured Ham. Critical Reviews in Food Science and Nutrition, 38(4), 331-352. http://doi.org/10.1080/10408699891274237 
Toldrá, F., Flores, M., \& Sanz, Y. (1997). Dry-cured ham flavour: Enzymatic generation and process influence. Food Chemistry, 59(4), 523-530. http://doi.org/10.1016/S03088146(97)00013-7

Warnants, N., Van Oeckel, M. J., \& Boucqué, C. V. (1996). Incorporation of dietary polyunsaturated fatty acids in pork tissues and its implications for the quality of the end products. Meat Science, 44(1-2), 125-144. http://doi.org/10.1016/S0309-1740(96)00029-0

Wood, J. D., Enser, M., Whittington, F. M., Moncrieff, C. B., \& Kempster, A. J. (1989). Wood et al., 1989 Backfat composition in pigs Differences between fat thickness groups and sexes..pdf. Livestock Production Science Elsevier Science Publishers B.V. Retrieved from http://ac.els-cdn.com/0301622689900663/1-s2.0-0301622689900663main.pdf?_tid=02bf6b6e-300f-11e7-a6c9-

00000aacb35d\&acdnat=1493822848_b5a764336449f25b0d2b04164c239dad 